\title{
UN MODELO PARA LA GESTIÓN DE LA INFORMACIÓN Y LA TECNOLOGÍA EN LA UNIVERSIDAD NACIONAL
}

\author{
Arthur Giesberts ${ }^{1}$ \\ Carlos Francisco Carranza ${ }^{2}$ \\ Rónald Miranda Chavarría
}

La Gestión de la Información, la Tecnología y las Comunicaciones (ITC) en una universidad pública no debe ser vista de manera mitificada como un asunto de especialistas; debe estar direccionada por las autoridades académicas y tener una correspondencia con el modelo de gestión: con el grado de desconcentración y con la rendición de cuentas. Debe desarrollar habilidades estratégicas en materia de información y tecnología paralos procesos académicos, de gestión y los servicios de apoyo. El proceso de gestión de la ITC comprende la planificación y organización, la adquisición y la puesta en ejecución de sistemas tecnológicos y de información, la entreg a y el soporte de servicios relacionados y el monitoreo. Se propone un modelo organizativo desconcentrado, con una instancia de asesoría a la Rectoría responsable de la información estratégica y de orientar el modelo de gestión de la información y la tecnología. Las vicerrectorías definen la naturaleza de la información requerida para los procesos académicos, de gestión y para los servicios de apoyo; en tanto que una instancia operativa administra las redes internas, los datos, el funcionamiento y la seguridad de los sistemas de información. El modelo que se presenta a continuación se ha propuesto para ser implementado en la Universidad Nacional, como parte de un proceso de mejora de los sistemas de generación, procesamiento y uso de la información en las actividades académicas y de gestión universitaria.
In public universities, information, technology and communications (ITC) management, is not to be considered an issue concerning experts only; it needs to be under the direction of academic authorities andmustconform with the management model of the institution, particularly with its degree of decentralization and the rendering of accounts. The model must develop strategic skills in information and technology matters for academic and management processes, and for support services. The ITC management process includes planning and organization, the acquisition and implementation of technological and information systems, the delivery and support of related services and, finally, a monitoring stage. We propose here a decentralized organization model that would include an entity for advisory assistance to the Rectorship, and which would be in charge of the strategic information and of guiding the information and technology management model. The vicerectorships will define the nature of the information required to feed academic and management processes, and support systems, while an operational entity manages internal networks, data and the functioning and safety of information systems. The model presented here is a proposal to be used at Universidad Nacional as part of an improvement process in generation systems, data processing and the use of these data in academic activities and university management.

1 Especialista en Gestión de la Información y la Tecnología. Instituto de Estudios para el Desarrollo (IVO), de la Universidad de Tilburg, Holanda.

2 Máster en Economía. Académico del Programa de Gestión de la Calidad Institucional. Universidad Nacional. Heredia, Costa Rica.

3 Máster en Adrninistración de Empresas. Director del Programa Gestión de la Calidad Institucional. Universidad Nacional. Heredia, Costa Rica. 


\section{Introducción}

Hoy más que nunca, en la sociedad globalizada y del conocimiento, el acceso oportuno a la información relevante y veraz otorga mayores ventajas que lo convierten en una de las más importantes fuentes de competitividad, del buen desempeño y de supervivencia de las organizaciones en el entorno social. La gestión de la información, como parte integral de un sistema de toma de decisiones y la gestión de la tecnología relacionada han estado en continua evolución, pero cada día, la velocidad de dichos cambios es mucho mayor que antes. La amenaza más importante a la cual podría enfrentarse una institución de educación superior, si no adopta de manera pronta y apropiada las medidas necesarias en estos asuntos, es perder su pertinencia social en el mediano y el largo plazo, como resultado de una incapacidad para disponer de la información oportuna que indique cuán productiva es la institución, cuán costosa es y cuál es el nivel de su calidad académica e institucional.

Este artículo aporta los elementos más importantes que deben ser considerados para cambiar el modelo de gestión de la información y la tecnología en una institución universitaria. La tesis fundamental que se plantea es que el tema de la gestión de la información y la tecnología es un asunto de vital importancia para la vida de una universidad, que no debe dejarse en manos de una instancia técnica especializada. Por el contrario, dada su importancia estratégica de la información para lograr mayores niveles de calidad en la actividad académica y de gestión universitaria, se requiere comprender el papel que la información y la tecnología cumplen en la actividad académica y en la puesta en práctica de un modelo de gestión institucional. Sobre ese fundamento es posible plantear una estrategia para la gestión de la información y la tecnología que se traduce a su vez en políticas y en una organización apropiada y coherente con los fundamentos del modelo de gestión, tales como la desconcentración y la rendición de cuentas. El modelo propuesto se plantea para el contexto de la Universidad Nacional de Costa Rica, en la cual la gestión de la información y la tecnología forma parte integral de un cambio en el modelo de gestión institucional.

\section{Objetivos de un Sistema de Gestión de la Información y la Tecnología}

El Sistema de Información y Tecnología se basa en dos referentes principales: uno, es una convención internacionalmente aceptada denominada "Objetivos de control para la información y tecnologías afines" conocido por sus siglas en inglés como COBIT (1998). Dicho referente establece la planificación y la organización de la información y la tecnología en función de los objetivos institucionales.

El segundo referente, es el modelo de gestión que se pretende desarrollar internamente en la Universidad Nacional, el cual en este caso, plantea tres premisas la desconcentración, la rendición de cuentas y la calidad institucional. 
Consecuentemente, el sistema de información y tecnología tiene como objetivo el posibilitar un buen funcionamiento de los procesos académicos y de gestión para que la institución en su conjunto cumpla con sumisión. Por ejemplo, facilitar el establecimiento y funcionamiento de un sistema de rendición de cuentas, requiere que los sistemas de información estén al servicio de las necesidades de la gestión para que se combinen los resultados académicos con los recursos asignados.

Análogamente, si el modelo pedagógico institucional contempla un uso más intensivo de la tecnología en los procesos de enseñanza-aprendizaje, los sistemas de información deberán poner en línea los recursos de información y de tecnología necesarios para cumplir con los objetivos académicos. En síntesis, los sistemas de información y tecnología deben ser eficaces, eficientes, apropiados y gestionados con sentido estratégico.

Es esencial la congruencia entre la estrategia institucional y la estrategia sobre la información y la tecnología. El Plan Global Institucional, en el cual se define la estrategia institucional de la Universidad Nacional, tiene por objetivo elevar su calidad y mejorar el alcance de la misión y la visión de esta casa de einseñanza. La estrategia institucional plantea que para el logro de la visión de futuro, es necesario el desarrollo de un conjunto de habilidades, entre ellas la de gestionar la tecnología, la información y las comunicaciones (Recuadro 1).

\section{Rerunation.}

Estrategia institucional "La estrategia de la Universidad Nacional consiste en asumir una posición de cambio integral, hábil e inteligentemente conducido, lo cual requiere que las decisiones institucionales estén predeterminadamente orientadas a desarrollar de manera distintiva" un conjunto de habilidades, entre las cuales está la siguiente:

\section{“El desarrollo de una gestión actualizada de la tecnología, la infor-} mación y las comunicaciones. Incorpora una concepción moderna y eleva al nivel estratégico la gestión de la información, la tecnología y las comunicaciones. Orienta el uso de la información y la tecnología de manera eficaz hacia la toma de decisiones, la producción académica y la eficiencia de los procesos internos. Involucra un concepto amplio de comunicación, imagen y mercadeo de los productos del quehacer universitario y la gestión del patrimonio documental que sirve a la academia. Prevé el fortalecimiento de un sistema de información riguroso, actualizado, completo y disponible".

Fuente: Universidad Nacional (2004). Plan Global Institucional 2004 - 2014. Heredia, pp: 34 y 40. 
Es importante reconocer que la información es un insumo necesario en el desempeño individual de las unidades académicas o administrativas, las facultades, las sedes, y así progresivamente hasta la institución como un todo. En el ámbito institucional se genera información que forma parte de sistemas mayores, i.e. el Sistema Universitario Estatal, el Sistema Nacional de Acreditación de la Educación Superior, el Sistema Fiscal Nacional, etc. Lo sustantivo del sistema de información obedece al propósito de contribuir a que todas las partes de la institución universitaria cumplan apropiadamente con la misión social y ćoadyuven al logro de la visión y la estrategia universitaria.

\section{Situación de la gestión de la Información y la Tecnología en la UNA}

Como una situación típica de muchas universidades públicas de América Latina, en el caso de la Universidad Nacional en Costa Rica, el papel de los usuarios ha sido muy limitado en la definición tanto de las políticas de información y tecnología como de los formatos de salida de la información. El Centro de Cómputo ha sido la instancia técnica que tradicionalmente ha definido las formas de procesamiento y la salida de información. Las políticas iniciales en este campo, se fueron generando de manera muy autónoma desde el Centro de Cómputo. No eran el resultado de una verdadera reflexión acerca de las necesidades y prioridades de las distintas unidades académicas ni de las instancias de administración.

Esto dio paso a planes relacionados con tecnologías informáticas formulados centralizadamente que luego dieron como resulatado una brecha sustantiva entre lo planeado y lo ejecutado. Un efecto crítico de esa modalidad de gestión fue la generación de una falta de sentido de pertenencia, de responsabilidad y de compromiso por parte de muchas autoridades, tanto académicas como administrativas, con respecto a la gestión de la información y la tecnología; consecuentemente, el tema quedó relegado a una instancia técnica.

Más recientemente se ha procurado una sinergia entre el Plan Global Institucional y la política de planificación de la tecnología y la información. También se ha procurado una mejor articulación con los planes de académicos y de gestión. El esfuerzo por introducir un concepto más integral y moderno de gestión de la información y la tecnología ha sido impulsado especialmente por el proyecto denominado UNAIS (Universidad Nacional Information System), que se ejecuta mediante la cooperación conjunta entre la Universidad Nacional y el Instituto de Investigación para el Desarrollo de la Universidad de Tilburg, Holanda (Giesberts, 2000). 
Como producto de un levantamiento exploratorio realizado en un taller con participación de autoridades institucionales, autoridades medias, tanto académicas como administrativas, representantes de equipos de apoyo relacionados con la información y la tecnología, académicos involucrados en programas de docencia o investigación con un uso intensivo de la información y la tecnología, se pudieron identificar los siguientes rasgos del panorama actual de la información y la tecnología en la institución.

En general, la planificación y la organización de la información y la tecnología se encuentra en un estadio inicial. Se tiene conciencia de la necesidad de contar con un plan estratégico de información y tecnología y con alguna dirección acerca del rumbo que debe tener la tecnología en la institución, así como algunos elementos de la arquitectura de la información o la necesidad de realizar inversiones en este campo. También se reconoce el papel de la información en la administración de la calidad.

En cuanto a la adquisición y la puesta en funcionamiento de la plataforma tecnológica se reconoce que aún no se cuenta con muchos procesos; sin embargo, se está en un nivel inicial en relación con la identificación de soluciones, la adquisición y el mantenimiento de la infraestructura técnica y la instalación de varios sistemas. Similar es el panorama relacionado con la entrega y el soporte. Aquí, los esfuerzos iniciales se orientan a preocuparse por garantizar la continuidad de servicios, especialmente de intranet, así como supervisar y asistir a los usuarios en el uso de la red; además, de la administración de los datos y de la configuración y la atención a problemas o contingencias relacionados con el uso de los sistemas.

En resumen, las mayores responsabilidades y esfuerzos se dan en la administración, el mantenimiento y la operación de los equipos de computación y las redes. Pero, ha prevalecido una carencia en el direccionamiento estratégico y en la definición del contenido de la información para guiar la toma de decisiones académicas y de gestión. Las relaciones entre la gestión superior, las decisiones, la información y la tecnología han sido históricamente débiles; de ahí la necesidad de buscar un modelo de gestión y organización de la información y la tecnología que corresponda con una concepción de desarrollo universitario estratégico y desconcentrado.

\section{Un marco estratégico necesario}

La definición de las necesidades y el direccionamiento de la gestión de la información y la tecnología parten del reconocimiento de los procesos primarios de la Universida: la docencia, la investigación, la extensión y la producción 
artística. Estos son los procesos que generan impacto social y que definen la pertinencia del trabajo institucional; además se ejecutan en las unidades académicas, centros e institutos de investigación y extensión. La información relacionada con la actividad académica debe dar cuenta de los productos tangibles de la institución (Sánchez, 2002): la cantidad de graduados, los aportes al acervo de conocimientos, las contribuciones específicas a los procesos de desarrollo del país y la producción artística. Esta información es fundamental para los informes que la Universidad brinde a los organismos gubernamentales y para demostrar su nivel de calidad académica. Otro tipo de información proviene de los denominados procesos secundarios de la institución. Estos involucran todo aquello relacionado con los recursos humanos, el manejo financiero y presupuestario, la logística, el mantenimiento, los servicios a los estudiantes, y el registro estudiantil, entre otros. Esta información permite medir el desempeño y la eficiencia de los responsables de los procesos de apoyo y su interrelación con los procesos primarios. Por ejemplo, se puede dar cuenta de cambios mensurables en el perfil intelectual y actitudinal de los estudiantes o su desempeño en las distintas escuelas. Para ilustrar más claramente, debe permitir observar cuántos estudiantes se mantienen o abandonan su carrera, y cuántos de ellos corresponden a los estudiantes meta de la Universidad.

El papel de la gestión superior de la Universidad consiste en definir los criterios y las formas con los cuales se deben conocer y presentar los diferentes tipos de información para evaluar el desempeño institucional, para verificar el grado de logro de la estrategia institucional, para la rendición de cuentas y, en consecuencia, para tomar las decisiones correspondientes con la direccionalidad institucional y con el uso de los recursos. Debe estar en capacidad de especificar el grado de agregación de la información requerida y definir los tipos de cruces o combinaciones que deben establecerse entre datos provenientes de la información primaria y los de la información secundaria. Estos son justamente los que permiten conocer el desempeño, tanto en productividad como en la calidad y la eficiencia en el uso de los recursos por parte de cada instancia de la organización. A manera de ejemplo, permite dar cuentas del costo de graduar a un estudiante en una carrera frente a otras o cómo ha evolucionado en los últimos años la productividad de los académicos de una facultad en términos de la cantidad de artículos publicados en revistas internacionales.

Para tener valor estratégico y utilidad, la información debe ser de calidad. Se considera de calidad cuando la información es completa, correcta, está disponible a tiempo y es autorizada. Su carácter estratégico lo determina su eficacia para hacer posible y dar cuenta del logro de la misión, la visión, las estrategias y las políticas en los distintos ámbitos de la institución. 
A partir de las definiciones de la gestión superior y de las de gestión funcional, tanto académicas (facultades) como administrativas (recursos humanos, finanzas, vida estudiantil, etc.) debe contarse con una dirección o unidad responsable de la gestión de la tecnología de la información la cual debe ocuparse de diseñar y dar mantenimiento al modelo de datos, definir los estándares, la nomenclatura, las herramientas de hardware y de software, administrar los servidores y las bases de datos. También debe responsabilizarse de la infraestructura tecnológica y garantizar su seguridad. La gestión específica en este ámbito debe conducir al establecimiento de una plataforma tecnológica más eficaz, más eficiente, que tenga soporte de mercado durante largo tiempo, que permita instalar la mayoría de los sistemas de información disponibles y que tenga el mayor acceso posible al peritaje suministrado por el mercado.

\section{El proceso de Gestión de la Información y la Tecnología}

Fundamentado en el modelo COBIT (1998) el proceso de Gestión de la Información y la Tecnología debe incluir una fase de planeación y organización, una fase de adquisición y puesta en ejecución de los sistemas de información y tecnología, una fase de entrega y soporte de dichos sistemas y finalmente una fase de monitoreo. Esas distintas fases se describen a continuación.

\section{Planeación y organización}

Un primer paso consiste en definir el carácter y el contenido del plan estratégico de información, tecnología de la información y comunicaciones (ITC), el cual debe estar debidamente enlazado con la planificación estratégica de la Universidad al más alto nivel institucional. Por tanto, las autoridades superiores, los decanos y los directores de servicios funcionales relevantes deben contribuir en la definición de los objetivos estratégicos que la institución busca con la gestión de la información y la tecnología.

El contenido del plan estratégico de información y tecnología debe contemplar las políticas de largo plazo (para períodos de 3 a 5 años) que deben regir a los distintos niveles de gestión institucional. También debe detallar los objetivos en plazos más cortos de uno o dos años, así como las actividades y el presupuesto requerido en cada año de vigencia del plan.

El plan de información y tecnología también debe facilitar el funcionamiento de todo el sistema de planificación institucional. Por ejemplo, los Planes Operativos Anuales deben tener una correspondencia con los planes estratégicos de mediano plazo, y deben incluir la información precisa que los convierta 
en un instrumento para el logro efectivo en el corto plazo de los esfuerzos y los resultados que apuntan a las esträtegias institucionales de mediano y largo plazo. Además, dicha información debe estar asociada con los resultados y los recursos sobre los cuales se rinden cuentas.

A partir de la concepción estratégica de la gestión de la información y la tecnología se establece un segundo paso: cómo debe estar organizada su gestión dentro de la Universidad. Esa organización debe corresponder a los tres niveles de gestión contemplados en el modelo de gestión y a su naturaleza desconcentrada. Es decir, define la función del nivel institucional o estratégico, las funciones de los niveles directivos en las facultades o servicios de apoyo y las funciones de las instancias operativas vinculadas con la tecnología y la información. Una propuesta relacionada con la organización institucional para la gestión de la información y la tecnología se presenta más adelante.

El tercer paso consiste en definir la arquitectura de la información, para lo cual se debe contar con políticas que permitan los siguientes logros:

- Proveer a los procesos académicos o primarios de la información requerida y de servicios de información y comunicación que agreguen valor a las actividades académicas. Para esto es importante establecer el grado de automatización de los procesos académicos.

- Usar adecuadamente los servicios de información y comunicación en los procesos secundarios, tales como finanzas, recursos humanos, servicios estudiantiles y la administración general.

Como parte de la definición de la arquitectura de la información, se establece cuál es la instancia responsable de proveer en forma agregada; es decir, aquella que sobrepasa el ámbito de competencia de las unidades particulares y que combina la información de unas instancias con otras; por ejemplo, la combinación de información estudiantil con la información financiera. La instancia responsable del manejo de este tipo de información debe depender del nivel gerencial de la institución y requiere de un equipo humano de alto nivel profesional, que sea capaz de procesar con oportunidad y calidad información gerencial.

El cuarto paso en la planificación estratégica de la información y la tecnología consiste en determinar la dirección tecnológica de la institución. Para eso se debe contar con las siguientes políticas:

- Monitorear cuidadosamente los productos tecnológicos para la información y las comunicaciones disponibles en el mercado, indagar las perspectivas de desarrollo para el futuro próximo y determinar el nivel y las opciones de uso que requiere la universidad. 
- En concordancia, se debe monitorear el desarrollo en la enseñanza, la investigación, la extensión y la gestión universitaria.

- Definir los requerimientos de información y de tecnología en los procesos primarios y secundarios y elaborar una propuesta para la puesta en ejecución de proyectos relacionados con la introducción o mejora de los sistemas de información, de tecnología y de comunicación.

\section{Adquisición y puesta en ejecución}

Para lograr una adquisición y puesta en ejecución de sistemas adecuados de información y de tecnología se debe contar con políticas para la adquisición de sistemas tecnológicos de información.

En el nivel superior o institucional las políticas proveen los criterios en cuanto a los proveedores, la tecnología, los aspectos contemplados en los contratos, tales como códigos fuente o actualizaciones, los servicios de soporte, el financiamiento (once off) y los derechos o licencias adquiridos.

A partir de las políticas se descentraliza la decisión de cuál sistema comprar. Esto contempla el análisis de la conveniencia de desarrollar sistemas propios frente a la posibilidad de compra de aplicaciones disponibles en el mercado que se ajusten a los requerimientos institucionales. Generalmente, este proceso se realiza con el concurso de un equipo interdisciplinario o un Comité de Gestión de Tecnología de la Información, compuesto por profesionales de la información y de la tecnología de la información, profesionales académicos de los campos de la docencia, la investigación, la extensión y la producción artística y profesionales de la gerencia universitaria. Este equipo debe identificar soluciones tecnológicas y recomendar la adquisición, la administración y el mantenimiento de aplicaciones de software, de infraestructura técnica, del desarrollo de procedimientos, de la certificación de los sistemas de información y de recomendar cómo se deben administrar los cambios que con el tiempo sea necesario introducir en los sistemas tecnológicos de información.

\section{Entrega y soporte}

Este tipo de proceso es responsabilidad de las instancias operativas conformadas por técnicos conocedores del manejo y el funcionamiento de los sistemas tecnológicos y de la información.

Se contempla aquí la infraestructura de los sistemas tecnológicos, los cuales deben responder a políticas que hagan posible que dicha infraestructura sea eficaz, eficiente y sostenible. Entre otros aspectos, las políticas deben: garantizar 
la seguridad de los sistemas de información, establecer cómo se administra la información, definir cómo se administra el uso de las instalaciones y la operación de los sistemas y cómo se identifican y asignan los costos de funcionamiento y mantenimiento.

Los administradores y otros técnicos que administran la infraestructura tecnológica se ocupan de la configuración, de asegurar la continuidad de los servicios, de los problemas operativos y de la alimentación de datos y la seguridad de los sistemas y de la información. También proveen los servicios técnicos como el mantenimiento y el entrenamiento de usuarios. Por tanto, deben definir los tipos y niveles de calidad del servicio que ofrece y administrar aquellos servicios que sean realizados por terceros o entidades externas.

\section{Monitoreo}

La contraloría y los auditores internos y externos deben comprobar el cumplimiento de los objetivos de control de los sistemas de información y las tecnologías relacionadas. De esta manera se puede tener constancia de que en la institución se cuenta con la definición de políticas claras y el empleo de prácticas seguras que son reconocidas internacionalmente por entidades comerciales, gubernamentales y profesionales (COBIT, 1998).

Es decisivo que el nivel superior de la institución defina las políticas y cuál será el sistema de control de la información y la tecnología que se utilizará en la institución, así como aclarar las responsabilidades que corresponden a las distintas dependencias en el proceso de control. El sistema de control debe incorporar aspectos como el ambiente de control, la valoración de los riesgos y las actividades que aseguran que la información y los sistemas están debidamente monitoreados y controlados (Delgado, 2002).

\section{Un modelo organizativo para la Gestión de la Información y la Tecnología}

La organización actual de la información y la tecnología ha seguido un patrón de desarrollo casuístico y coyuntural que ha dado origen a la existencia de múltiples actores involucrados, pero con formas de trabajo desarticulado. Es por eso que se requiere de un proyecto de reorganización de la arquitectura de la Gestión de la Información y la Tecnología que concentre instancias dispersas y establezca las competencias y dinámicas de las distintas instancias (Alfaro y Ortiz, 2000).

El modelo de organización propuesto adopta como referente de diseño al modelo de gestión institucional, el cual se orienta a la calidad; contiene un enfoque estratégico, es desconcentrado y requiere de un sistema de rendición de cuentas (Figura 1). 


\section{Modelo de organización para la gestión desconcentrada}

de la información y la tecnología

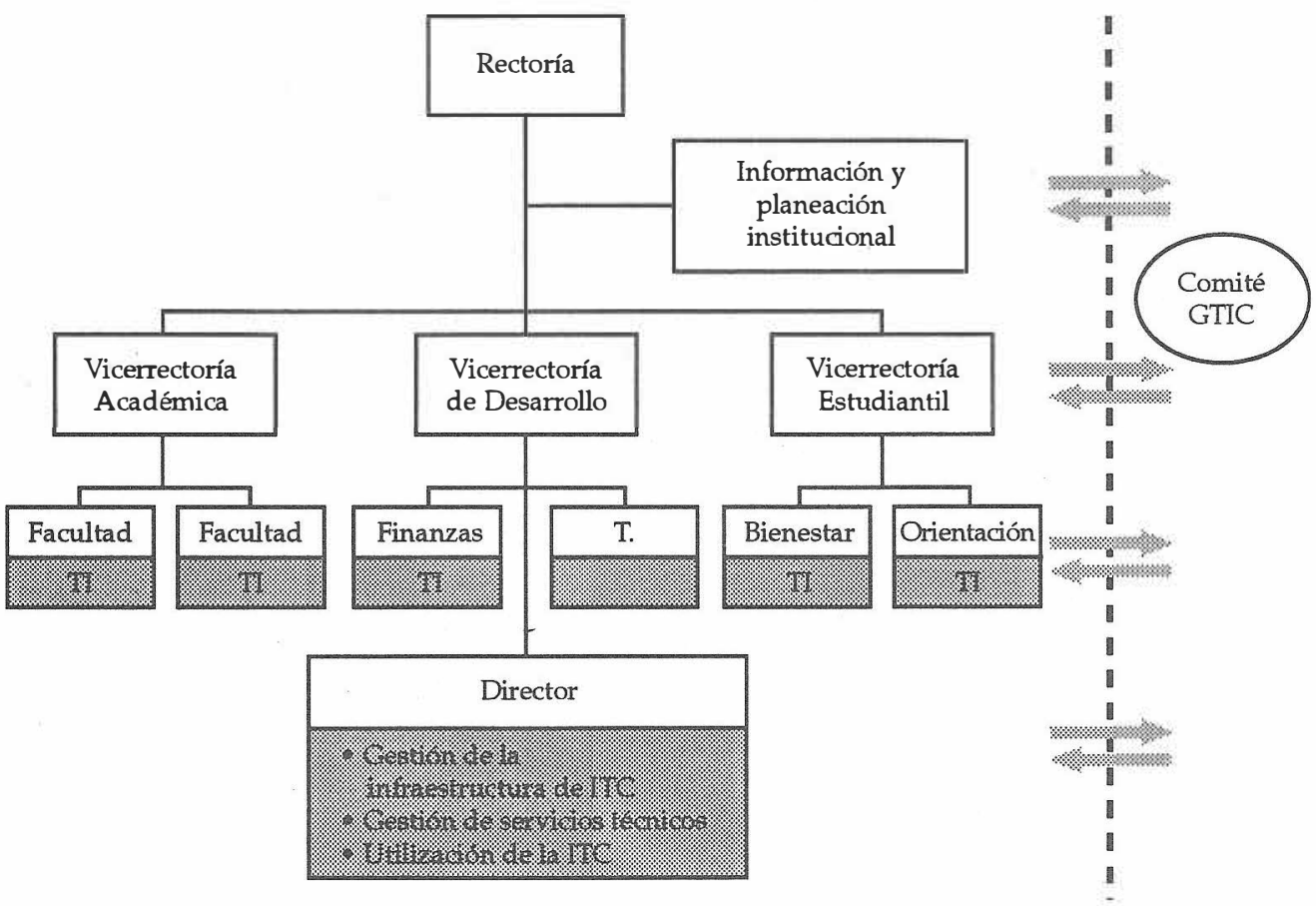

Figura 1. Modelo de organización para la gestión desconcentrada de la información y la tecnologia. Las áreas en gris representan la función especifica relacionada con la gestión de información y tecnología en las instancias funcionales. Las áreas en blanco representan las funciones generales de gestión, de apoyo o académicas.

En primer lugar, la Rectoría debe reconocer y estar en capacidad de cumplir a cabalidad su rol para lograr que la información y la tecnología tengan el lugar que permita hacer de la institución una universidad competitiva. Para eso, requiere de una instancia que forme parte de la tecnoestructura de la organización en la que se concibe la manera de realizarel proceso y el contenido de la planificación estratégica institucional y la manera de gestionar la información institucional. Esta instancia asume la responsabilidad de la concepción y facilitación de la elaboración de los futuros planes de gestión de la información y la tecnología.

Es importante la existencia de un comité institucional de gestión de la información y la tecnología constituido por personas competentes y representativo de los diferentes niveles de gestión. Debe contar con una persona que lo preside y todos sus miembros deben tener una clara comprensión del modelo de gestión y de organización de la información y la tecnología. El comité se ocupa de observar y analizar críticamente la gestión de la información y la tecnología en la institución; advierte acerca de los problemas que se presentan en los distintos niveles y recomienda estrategias y políticas que debe adoptar la universidad. 


\section{La eficacia y la eficiencia finales} de la Gestión de la Información y la Tecnología dependen tanto de la definición asertiva de las políticas por parte de las autoridades universitarias como de las capacidades para elegir y mantener en buen

funcionamiento los sistemas
tecnológicos y de información
con los atributos más adecuados
para la Universidad.

Las vicerrectorías son las responsables del direccionamiento de la gestión de la información en el ámbito funcional correspondiente. Así, en lo académico se establecen los requerimientos de información por parte de las facultades para cumplir con las funciones de docencia, investigación, extensión o producción; o bien en los servicios estudiantiles se establecen las necesidades de información de los programas socioeconómicos, de orientación estudiantil, del registro estudiantil o de los centros de documentación y bibliotecas. En los temas relacionados con el desarro-

llo instucional, se establecen los requerimientos de información para el eficaz desempeño de la gestión del talento humano, las finanzas o los servicios de logística, entre otros. Estas necesidades de información se desprenden de las estrategias funcionales de desarrollo de cada área en función de la estrategia institucional. Estas instancias deben contar con acceso a la información estratégica y académica de la red mundial, difundir sus resultados por medio de dicha red, contar con las facilidades para procesar la información pertinente y todo el soporte de información para cumplir con las funciones académicas, científicas y culturales de la institución.

Como parte de la Vicerrectoría de Desarrollo se establece la instancia operativa responsable de la administración de la red interna de información, así como de toda la infraestructura correspondiente: los servidores, las redes físicas o inalámbricas, los sistemas operativos, la administración de las bases de datos, las impresoras, centrales telefónicas y cualquier otro equipo requerido para el buen funcionamiento de los sistemas de información. Como parte de la administración de los datos, le corresponde certificar que los datos suministrados por medio del sistema de información sean documentados, transparentes y replicables. Esta instancia también es responsable por la administración y el mantenimiento de la página web institucional y establece las normas técnicas relacionadas con su uso, diseño y actualización. Se ocupa de la coordinación de los servicios de información y comunicación que incluyen a la biblioteca y otras instancias relacionadas con las telecomunicaciones. Además, provee los servicios centralizados 
como el mantenimiento de la plataforma tecnológica y el entrenamiento de usuarios. El director de esta instancia no debe ser un técnico, sino un profesional capaz de entender y priorizar las demandas académicas, de las autoridades universitarias y de las instancias funcionales de apoyo.

El apoyo técnico, el mantenimiento de los equipos y las actualizaciones de software en línea de acuerdo con las licencias y los contratos, se gestiona de manera desconcentrada. El personal técnico se destaca en las distintas vicerrectorías, facultades y sedes, lo cual asegura una atención oportuna a los distintos usuarios. Los funcionarios técnicos destacados en las instancias desconcentradas deben mantener una relación constante con las instancias central para efectos de garantizar el cumplimiento de las políticas institucionales acerca de la información y la tecnología.

\section{Consideraciones para la puesta en ejecución}

La ejecución de una estrategia y una nueva política de planificación de las tecnologías, la información y las comunicaciones comporta una alta capacidad para poner en práctica decisiones, nuevas normas y la fijación de metas concretas.

Es importante que exista una visión total del presupuesto y gastos realizados en información, tecnología y comunicación. Esto debe hacerse considerando que los planes institucionales y académicos contienen actividades relacionadas con la política de planificación de la información y la tecnología y que los planes académicos contienen presupuesto para las actividades de la política de planificación de la ITC.

Por su parte, los departamentos poseen la responsabilidad de planificar sus actividades y presupuestos, contemplando las necesidades y desarrollos requeridos en cuanto a información y tecnología. También son responsables de la realización de las metas concretas y del cumplimiento de las normas institucionales.

Hay elementos de la política acerca de información y tecnología que requieren del cruce de información entre instancias diferentes o de la ejecución de proyectos que requieren de las contribuciones de varias instancias; por ejemplo, el desarrollo de un ambiente de aprendizaje digital. Este tipo de esfuerzos revelan la necesidad de un gran esfuerzo de coordinación y a la vez, de entender que la política de gestión de la información y la tecnología debe crecer y madurar a lo largo y ancho de la institución universitaria.

Una consideración final es la conveniencia y la necesidad de introducir un proceso de capacitación en gestión de la información y de las tecnologías dirigido expresamente a las autoridades universitarias, tanto de la gestión superior como de las facultades. El propósito es desmitificar la gestión de la información 
y la tecnología para lograr una plena comprensión del rol que deben asumir todas las autoridades académicas de la universidad. Para eso es importante el conocimiento de fundamentos, estrategias y herramientas que potencian el desarrollo de una institución académica a partir de una acertada integración de la gestión de la información, la tecnología y las comunicaciones.

\section{Conclusión}

La gestión de la información, la tecnología y las comunicaciones en un ambiente universitario contemporáneo requiere de un replanteamiento importante relacionado con el modelo de gestión y de desarrollo académico de la Universidad. Las autoridades universitarias deben estar en capacidad de establecer definiciones y políticas que propicien una gestión estratégica de dichos procesos.

Un nuevo modelo de gestión de información y tecnología implica la comprensión del modelo de gestión deseado para la Universidad, del papel de la información y la tecnología en los procesos de docencia, investigación y extensión; así como del papel de los servicios de apoyo a la academia y de las necesidades de información de la gestión superior. Es entonces cuando se cuenta con los elementos para proponer un plan de gestión de información y tecnología, cuya puesta en ejecución contempla una reorganización de las instancias y procesos relacionados.

La eficacia y la eficiencia finales de la gestión de la información y la tecnología dependen tanto de la definición asertiva de las políticas por parte de las autoridades universitarias como de las capacidades para elegir y mantener en buen funcionamiento los sistemas tecnológicos y de información con los arributos más adecuados para la Universidad.

Finalmente, se debe tener presente que la puesta en ejecución de una estrategia de información y tecnología requiere del aprestamiento y aseguramiento de las condiciones de logro necesarias: presupuestarias, organizativas y de capacidades. Esto último, significa elevar las capacidades de las autoridades universitarias para desempeñar adecuadamente su papel en la Gestión de la Información y la Tecnología.

\section{Referencias}

Alfaro, M. y Ortiz, P. (2000). Desarrollo de recomendaciones para la implantación de un Plan Estratégico Institucional para la Tecnología y la Información en la Universidad Nacional. Heredia. Maestría en Administración de Tecnología e Información.

COBIT. (1998). Objetivos de control para la información y tecnologías. Resumen ejecutivo. México. Information Systems Audit and Control Foundation. 
Delgado, X. (2002). Seminario-taller: Objetivos de control para la información y tecnologías afines "COBIT". Heredia. D.R.

Giesberts, A. (2000). El proyecto UNAIS. Tilburg. Development Research Institute.

Sánchez, V. (2002). Gestión de la información. Heredia. Universidad Nacional.

Universidad Nacional. (2004). Plan global institucional 2004-2012. Heredia.

Universidad Nacional. (2002). Un Concepto de Gestión para la Universidad Nacional. Heredia. 